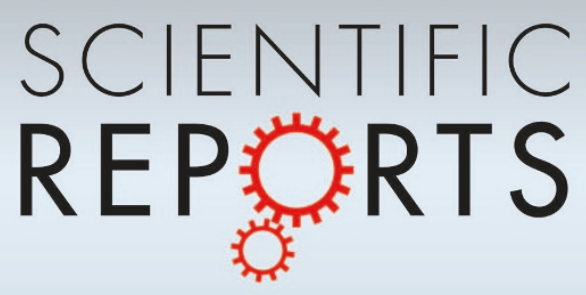

OPEN

SUBJECT AREAS:

PLANT BIOTECHNOLOGY

MUCOSAL IMMUNOLOGY

RISK FACTORS

MICROBIAL GENETICS

Received

27 December 2012

Accepted

28 May 2013

Published

11 June 2013

Correspondence and requests for materials should be addressed to K.L.H. (hkl009@163. com) or W.T.X. (xuwentaoboy@sina. com)

\section{Effects of genetically modified T2A-1 rice on the Gl health of rats after 90-day} supplement

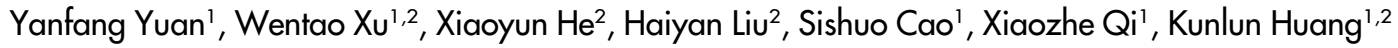 \\ \& Yunbo Luo',
}

'Laboratory of Food Safety, College of Food Science and Nutritional Engineering, China Agricultural University, Beijing 100083, China, ${ }^{2}$ The Supervision, Inspection \& Testing Center of Genetically Modified Food Safety, Ministry of Agriculture, Beijing 100083, China.

Bacillus thuringiensis insecticidal toxin (Bt) rice will be commercialized as a main food source. Traditional safety assessments on genetically modified products pay little attention on gastrointestinal (GI) health. More data about GI health of Bt rice must be provided to dispel public' doubts about the potential effects on human health. We constructed an improved safety assessment animal model using a basic subchronic toxicity experiment, measuring a range of parameters including microflora composition, intestinal permeability, epithelial structure, fecal enzymes, bacterial activity, and intestinal immunity. Significant differences were found between rice-fed groups and AIN93G-fed control groups in several parameters, whereas no differences were observed between genetically modified and non-genetically modified groups. No adverse effects were found on GI health resulting from genetically modified T2A-1 rice. In conclusion, this study may offer a systematic safety assessment model for GM material with respect to the effects on GI health.

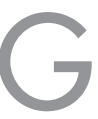
enetically modified plants expressing insecticidal traits offer a new strategy for crop protection, but at the same time, present a challenge in terms of food safety assessment. The Bacillus thuringiensis insecticidal toxin $(\mathrm{Bt})$ gene, which includes a range of different Cry genes, expresses gram-positive spore-Bt toxins (Cry proteins) that have been used in insect-resistant biotechnology for many years and have a long history of safe use $^{1,2}$. There are many types of commercially-grown Bt products used around the world including $\mathrm{Bt}$ corn, $\mathrm{Bt}$ cotton, Bt canola and Bt potatoes. Bt rice is only semi-commercialized in Iran, the only transgenic rice approved for commercial production are three herbicide (glufosinate)-resistant lines used in the United States ${ }^{3}$. In late 2009, China's Ministry of Agriculture released biosafety certificates for the Bt rice Huahui No. 1 and Bt Shanyou 63. Other field trials on Bt rice have also been conducted in Pakistan, Spain, Iran and India. Reports of the development and evaluation of Bt rice have been reviewed ${ }^{4}$. Biotech crops have already been demonstrated their capacity to significantly increase productivity and income. So they can contribute to the alleviation of poverty for the world's resource-poor farmers during a global financial crisis. It is true clear that the potential of the crops like Bt rice for the future is enormous ${ }^{5}$. Bt rice is the most promising genetically modified (GM) rice for commercialization. However, public concern over its safety is a major barrier to its release ${ }^{6}$.

For the last ten years, in accordance with the increased use of GM foods for human and livestock, a large number of feeding studies have been carried out on Cry proteins, which are regarded harmless or non-toxic to mammals ${ }^{1,7,8}$, including humans ${ }^{9}$. The innocuity may be due to the acidified gut pepsinolysis and the lack of Cry protein binding sites on mammalian gut epithelial cells ${ }^{10}$. However, it has been reported that Crylab is able to bind to the intestinal mucosal surface, influencing some epithelial cell functions ${ }^{11}$. Some minor changes on the distal intestine ${ }^{12}$ and mid-intestine of salmon have also been found. Although extensive in vitro-based studies have confirmed that the Bt protein disappears quickly in the stomach and other results provided evidence that the protein could persist in the gut and feces of animals, due to a food protection mechanism ${ }^{13,14}$. Until now, only sparse information regarding the unintended effects of genetically modified organisms (GMO) on gastric health has been available.

The intestinal tract is not only the first site contacting with the environment, bacteria and food antigen but also the largest immune site in the host and has important functions in metabolic reactions, nutrition absorption and regulation of immunity ${ }^{14,15}$. The relationship between gastrointestinal (GI) health and host disease has been 
seriously studied ${ }^{14,15}$. Besides, an incresing number of studies about the relationship between health and probiotics emerge in the food field $^{16}$. However, in GM product assessment, classical methods for analyzing the potential toxicological risk of a GM plant as whole food involves blood and urine chemistry, organ weight assessment and gross histopathological examination, with less focus on gastric health. It has also been suggested to look for specific physiological biomarkers of early ill effects to increase the diagnostic value and sensitivity of toxicity tests on food ${ }^{17}$. Although there is no evidence of any harmful effects related to GM crops or products, it is essential that genetic products be subjected to detailed safety assessments, including possible unintended effects before entering into the market $^{18}$.

Unintended changes result from the random insertion of a transgene into the host genome, which might disrupt endogenous gene expression and thus, result in changes of macro- or micronutrients, anti-nutritional factors or other indexs. Such changed factors might be toxic at low levels or could be potent allergens to the individuals ingesting GM food ${ }^{17}$. Targeted analysis designed to look for these types of changes are biased and will not cover all known and unknown compounds in the GM plant. Untargeted methods, like genomics, proteomics and metabolomics, have facilitated the analysis process. However, the lack of reference databases for the interpretation of complicated data prevents the acceptance of these methods as part of a routine strategy for safety assessment. Furthermore, the known assessments of the unintended effects of GM materials scarely involved animal experiments, much less GI health. Additionally, the lack of standardized methods for the evaluation of unintended effects may also be a reason for the limited research in this area. Another major concern for unintended effects is the potential migration of exogenous transgenes from GM plants to humans or animal intestinal microbes ${ }^{19,20}$. To date, there have been no reports demonstrating the transfer of exogenous genes to tissues or intestinal microbes of animals ${ }^{14,21,22}$. However, evidence of gene transfer from GM-soya to gut microorganisms was found in mixed cultures of human intestinal bacteria ${ }^{23}$. Related reviews have been published $^{23-25}$.

Although there are no reports demonstrating adverse effects of $\mathrm{Bt}$ rice on human or animal health, case-by-case assessments of GM events as the standard in both Norway and the $\mathrm{EU}^{26}$ have been advocated and adopted. Because different GM plants express different transgenic proteins and potentially have different unintended changes. Recent studies have also shown the effects of GM rice on the intestinal microflora of rats $^{27,28}$. Conclusions from these studies are superficial and controversial due to insufficient data reflecting gastric health. The GI system is complicated, which is affected by many interactive factors like $\mathrm{pH}$ value, composition of the microflora, metabolic products (e.g., fecal output and short-chain fatty acids (SCFAs)), the immune system, antioxidant activity, and epithelial structure ${ }^{15}$. It is widely accepted that gut flora might also be an essential factor in certain pathological disorders including multisystem organ failure, colon cancer, and inflammatory bowel disease ${ }^{29}$.

Rice is the most important food crop in the world, which feeds half of humanity and is especially important for the poor ${ }^{18}$. In China, about 800 million people take rice as main food. More than $90 \%$ of the world's rice is produced and consumed in Asia ${ }^{30}$. The present 90day feeding study was designed to assess the unintended effects of T2A-1 rice on the GI health of rats, using a series of parameters, including toxicity, immunological, and metabolic parameters, in order to establish a model for a systematic safety assessment of genetically modified products on GI health.

\section{Results}

No adverse effects were found on general health. Throughout the study, no signs of adverse effects were observed in the clinical appearance of the rats. No intestinal disturbances, like diarrhea or vomiting, were observed. Body weight and food intake were measured and calculated weekly, the results of which illustrated normal and similar growth patterns (Fig. S1-A) and no differences on weekly food intake (Fig. S1-B) among all the groups. No difference (Table S1) was found in either absolute weights or relative weights of selected organs between the GM and non-genetically modified (NM) groups. The only difference between the GM groups and NM groups was that Mean Corpuscular Hemoglobin $(\mathrm{MCH})$ value and Mean Corpuscular Hemoglobin Concentration (MCHC) value of NM group were $5.4 \%$ and $4.3 \%$ respectively higher than that of female GM group (Table S2). With respect to blood biochemistry (Table S2), no differences were found between the GM and NM groups. Several sex-dependent differences existed between rice-fed groups and the AIN93 G group, which are within the normal reference interval for rats of this breed and age $e^{10,28,31}$. Thus, none of the results was considered to be adverse. In another 90-day study, higher concentration of urea and a reduction in the concentration of protein were reported in male rats fed with $\mathrm{Bt}$ rice ${ }^{10}$. In this study, parameters like aspartate transaminase (AST), alanine transaminase (ALT) (reflecting liver function) and urea nitrogen (reflecting kidney function) did not differ among the groups. In conclusion, no adverse effects were observed.

Comparable differences were observed in the bacterial composition from GI tract content. The results of the bacterial quantification, based on real-time quantification PCR targeting the 16S rDNA of bacteria, are shown in Table S3. No difference $(\mathrm{P}>0.05)$ was found among each group in the measured bacteria and total bacteria in feces at 60 and 90 days post-treatment, but when compared with the AIN93 G group, 70\% rice control groups displayed increased numbers of Lactobacillus (NM: Male, $\mathrm{P}=0.028$, Female, $\mathrm{P}$ $=0.043 ; \mathrm{GM}$, Male, $\mathrm{P}=0.048$, Female, $\mathrm{P}=0.031$ ) and Enterococcus (NM: Male, $\mathrm{P}=0.023$, Female, $\mathrm{P}=0.007$; GM, Male, $\mathrm{P}=0.024$, Female, $\mathrm{P}=0.022$ ) in feces at 30 days post-treatment. However, no statistical difference was observed between the GM and NM groups. This tendency declined in the feces at 60 day post-treatment and disappeared at 90 days post-treatment, except that higher amounts of Enterococcus ( $\mathrm{P}>0.05)$ were still displayed in the rice-fed group compared to the AIN93 G group at 90 days post-treatment. Further analysis of relative abundance of the selected bacteria showed that three main probiotics (Lactobacilli group, Bifidobacteria genus, and Bacteroids) in feces accounted for $33.6 \%-47.4 \%$ of total bacteria, whereas the conditional pathogens, including Escherichia coli subgroup, Enterococcus genus, and Clostridium perfringens, only made up less than $1 \%$ of the total bacteria during all stages of the experiment. The ratios of $\mathrm{B} / \mathrm{E}$ (Bifidobacterium/Enterococcus), with a range from 1.19 to 1.33 , showed poor changes during the entire experiment stages and among each group, which indicated a good balance of gastric health. This result was consistent with a published study in which no significant influence of Bt corn could be found on the composition of the microbial population in the rumen of cattle ${ }^{14}$. With respects to the duodenum and ileum, no differences in bacterial composition were found, while significantly increased Lactobacillus and Enterococcus were observed in cecum contents on 90 days posttreatment. A recent study involving the feeding of Cry $1 \mathrm{Ab}$ protein to rats showed reduced amounts of Bifidobacterium in the duodenum and increased amounts of Coliforms in the ileum, but no changes on fecal bacterial counts ${ }^{9}$. In another $60 \%$ Galanthus nivalis (GNA) feeding experiment ${ }^{27}$, results showed statistically significant increases of total anaerobic bacteria, Lactobacillus and Enterococcus in duodenum samples, whereas decreased Enterobacteria in ileum samples were observed in the GM group $(\mathrm{P}<0.05)$.

Molecular analysis by amplification of $16 \mathrm{~S}$ rRNA genes and denaturing gradient gel electrophoresis (DGGE) was performed on samples from six individuals of each group. Each rat had a unique microbiota composition with a different band profile (Fig. 1). The 
A

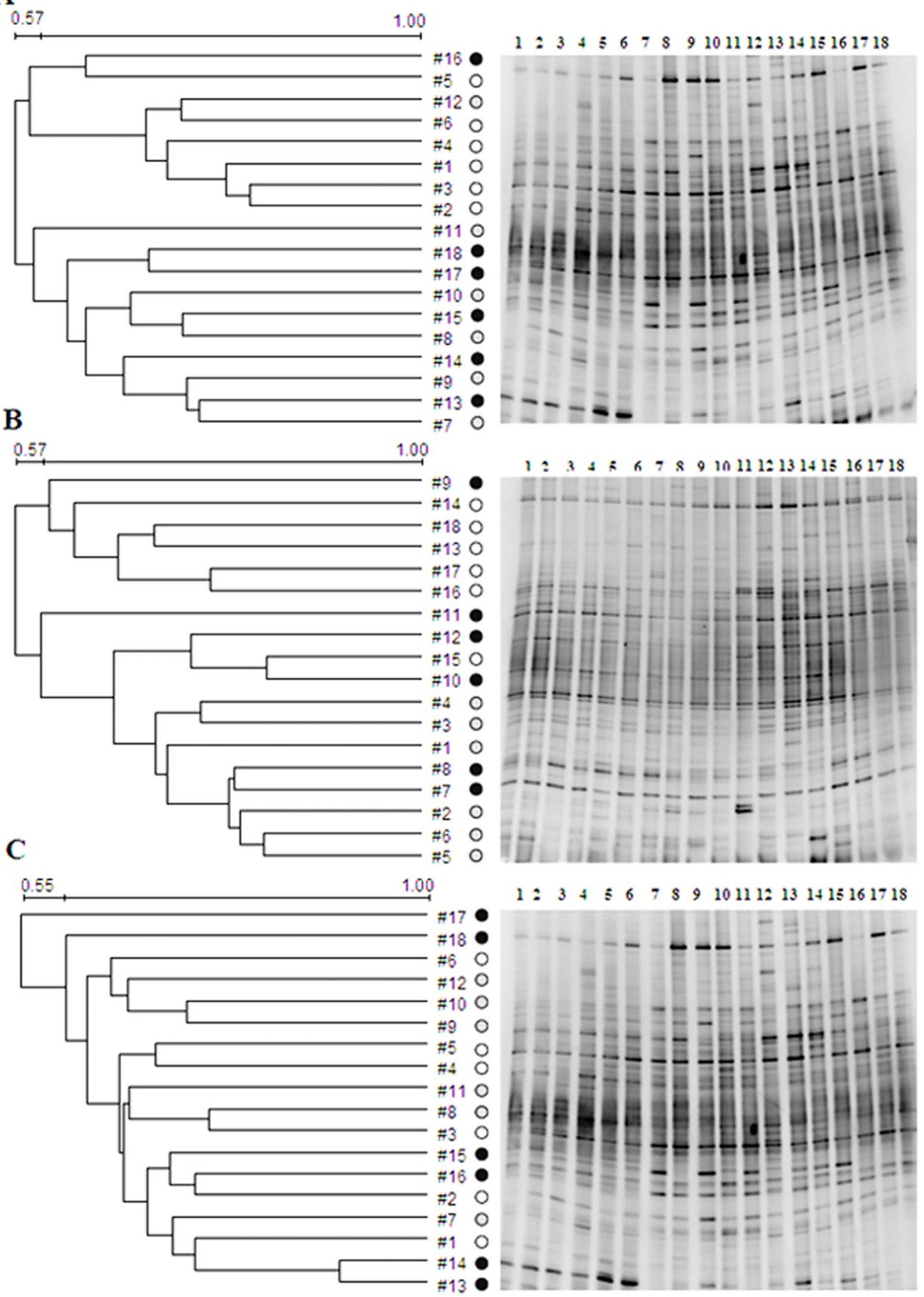

Figure 1 Results of denaturing gradient gel electrophoresis (DGGE) analysis by UPMGA. DGGE fingerprint patterns of microbial community from selected samples of six rats/group were performed with (A) feces at 30-day post-treatment, (B) content from cecum and (C) feces at 90-day posttreatment. O: AIN 93 G group; O: non-genetically modified (NM) group; $O$ : genetically modified (GM) group. 
Table 1 | Effect of Cry2A rice treatment on fecal biotransforming enzymes in rats

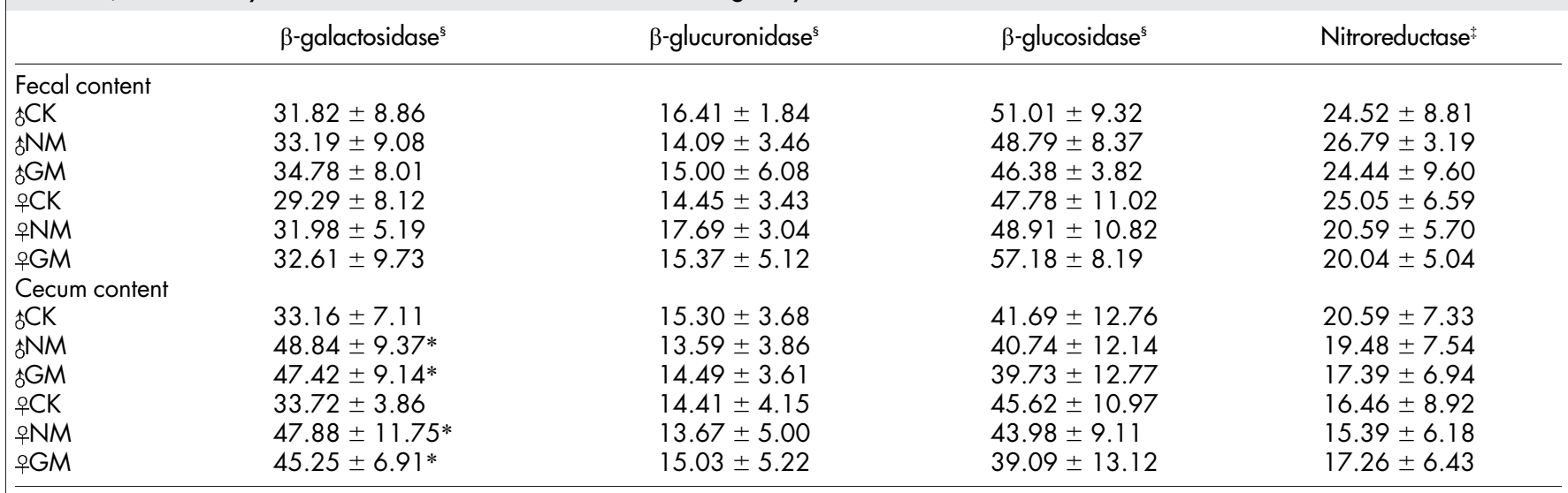

Values are mean \pm SD of eight rats from each group.

*: Statistically significant differences from the CK group $(p<0.05)$

§: Milligrams of $\beta$-nitrophenol liberated $/ \mathrm{min} / \mathrm{g}$ protein

t: Micromoles of $\beta$-aminobenzoic liberated $/ \mathrm{min} / \mathrm{g}$ protein.

effects of dietary treatments on bacterial composition were also clearly distinguished by the cluster analysis method. DGGE analysis showed two distinct groups (the rice diet group and AIN93 G group) with both fecal content after 30-day treated and cecum content after 90-day treated (Fig. 1-A, B). Results from the feces of 90-day treated rats (Fig. 1-A, C) exhibited similar band diversity in each group and indicated an increase in microbial diversity when compared to the feces of 30-day treated rats. The DGGE patterns reflected the predominant bacteria in the cecal samples, which reflected major changes induced by the different diets.

Small effects on microbiology activities existed. The microbial breakdown of carbohydrates is an anaerobic process known as fermentation, which yields SCFAs including acetic, propionic, and butyric acids as main products. The content of SCFAs is mainly affected by food sources and the bacteria composition in gastrointestinal tract (GIT). These bacterial metabolic products have great effects on host health, especially butyric acid output, which has been shown to have negative relationships with colon cancer and other diseases. In this experiment, acetate, propionate and butyrate decreased dramatically from the cecum to feces in all groups. Both GM and NM rice-fed groups displayed similar bacterial cellular fatty acid profiles in feces as AIN93 G group, but got a higher acetate output in cecum content than AIN93 G group (Table S5). NM and GM groups have lower $\mathrm{pH}$ values compared to $\mathrm{CK}$ groups in cecum content but the trend didn't take place in feces(Table S4). Although it is unknown that why intestinal acidity increases, the consumption of Cry2A rice had no adverse effects on SCFA production in the GIT of rats. No differences were found between the GM and NM groups in the composition of SCFAs profile of both feces and cecum. Results from a three-year feeding study of Bt176 maize on ovine supported our findings ${ }^{32}$, which showed that there was no change on the $\mathrm{pH}$ value and content of volatile fatty acids in luminal contents. Intestinal bacteria could play a part in the initiation of colon cancer through the production of carcinogens, cocarcinogens or procarcinogens, with the production of some enzymes ${ }^{29}$. In this experiment, the changing of diets did not have effects on the bacteria enzymes $\beta$ glucuronidase, $\beta$-glucosidase, and nitroreductase in feces (Table 1 ), but an increased amount of $\beta$-galactosidase in the cecum was observed in the GM and NM rice-fed groups, which supported the results of higher amounts of Lactobacillus in this part of GI tract. Other results showed no significant differences on weights and water content (Table S5) in cecum contents and feces among each group.

No damages were observed on intestinal permeability and mucosal immunity. Intestinal permeability was measured in the $70 \%$ rice-fed rats. Female rats exhibited similar absorption tendencies of DX-4000-FITC in plasma (Fig. 2-A) and diamine oxidase (DAO) levels in serum (Fig. 2-B). Furthermore, RT results (Fig. 3-A, B) and immunohistochemical results (Fig. S2) showed poor changes on expression of zonula occluden 1 (ZO1) and occludin proteins between the GM and NM groups. Close attention has been paid to
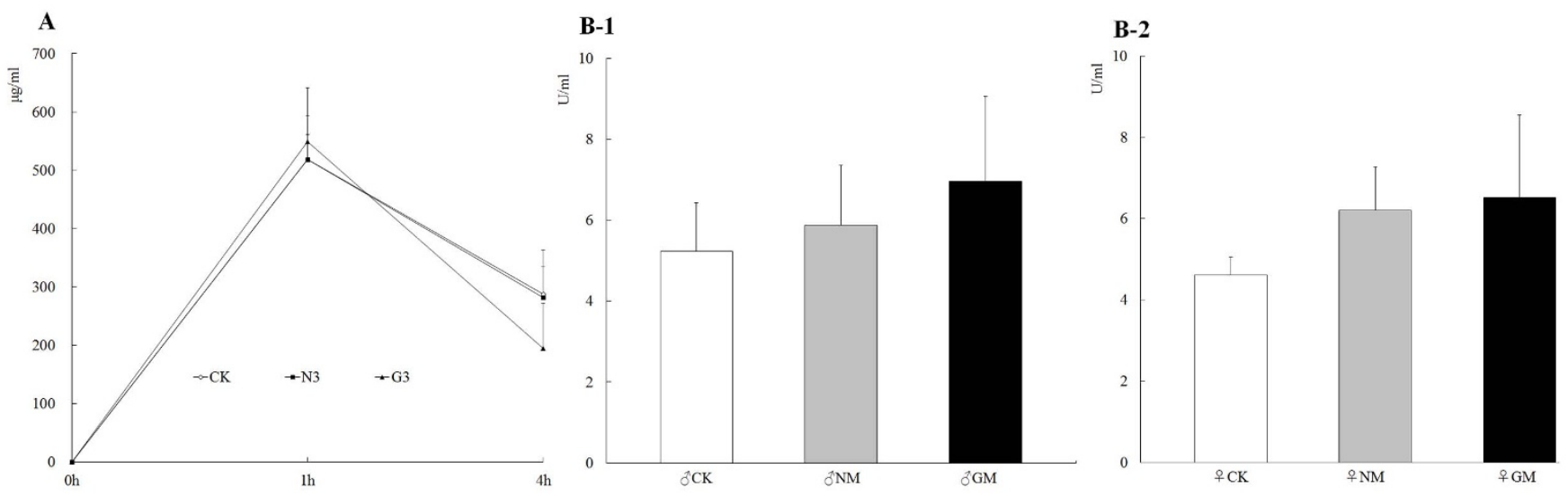

Figure $2 \mid$ Changes on mmbrane filter in gut mucosa. Membrane filter changes in gut were measured by $(A)$ FITC-4000 in plasma, $(B-1)$ diamine oxidase $(\mathrm{DAO})$ in serum of male rats and $(B-2) \mathrm{DAO}$ in serum of female rats. 
A-1

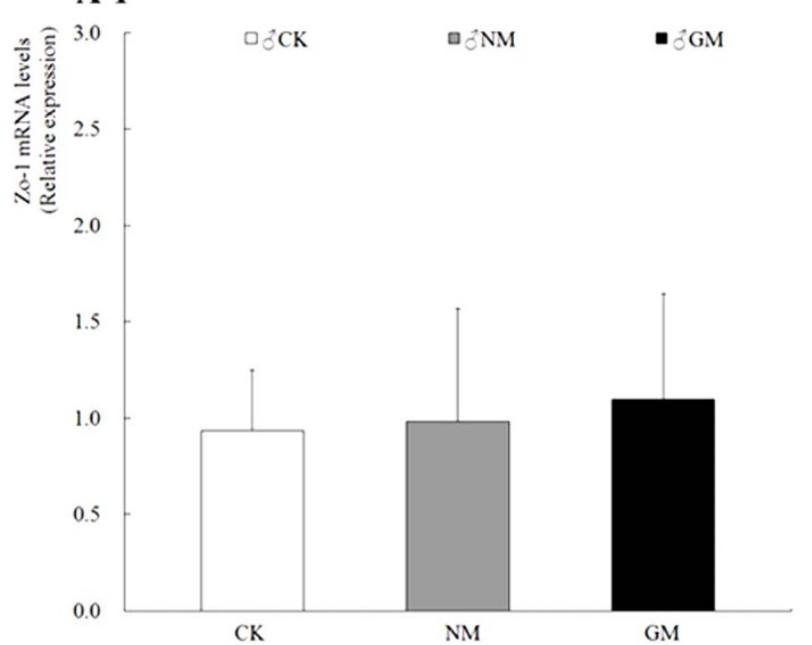

B-1

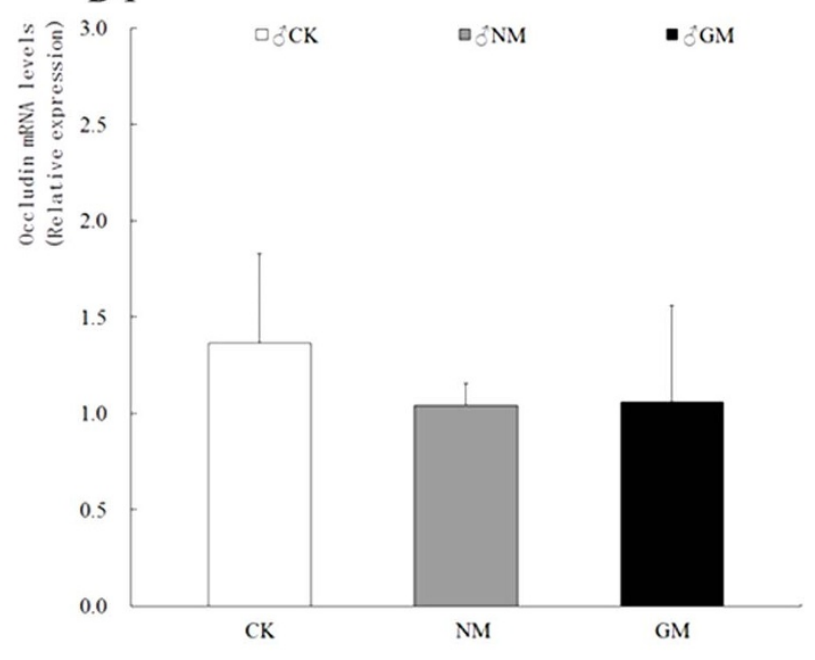

$\mathrm{C}-1 \quad \square \mathrm{CK} \quad \square$

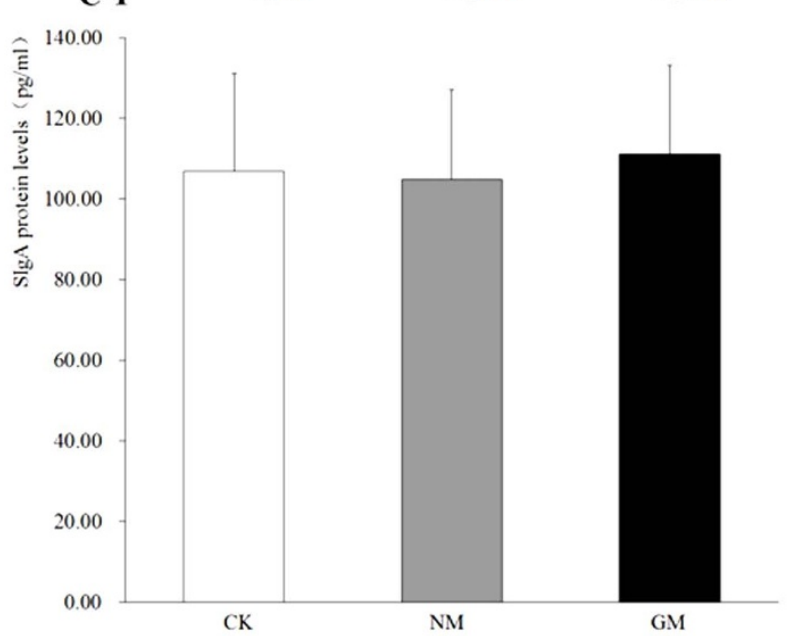

A-2

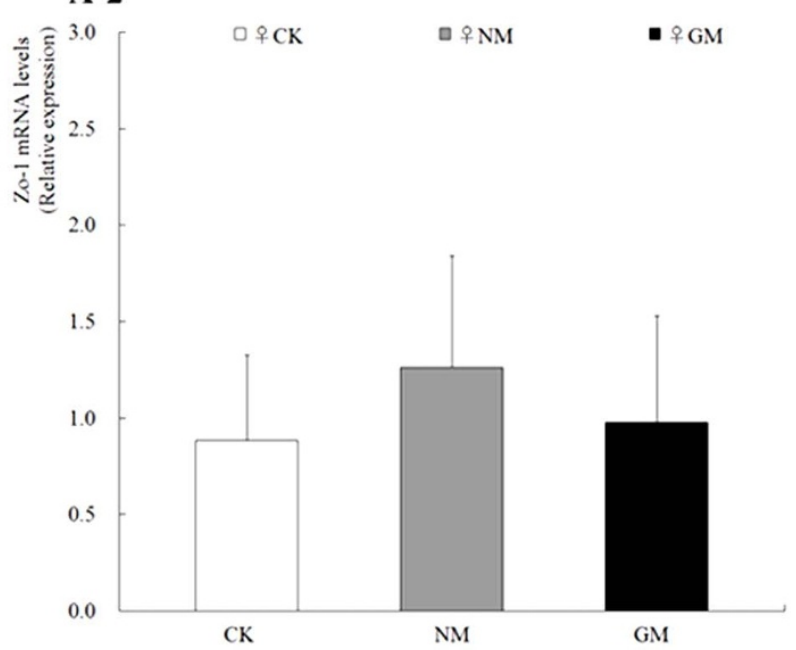

B-2

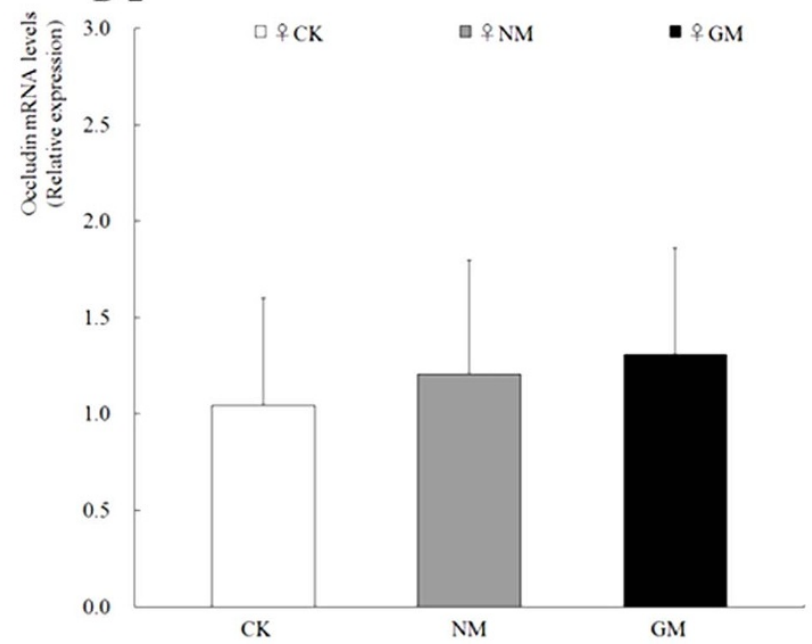

GM

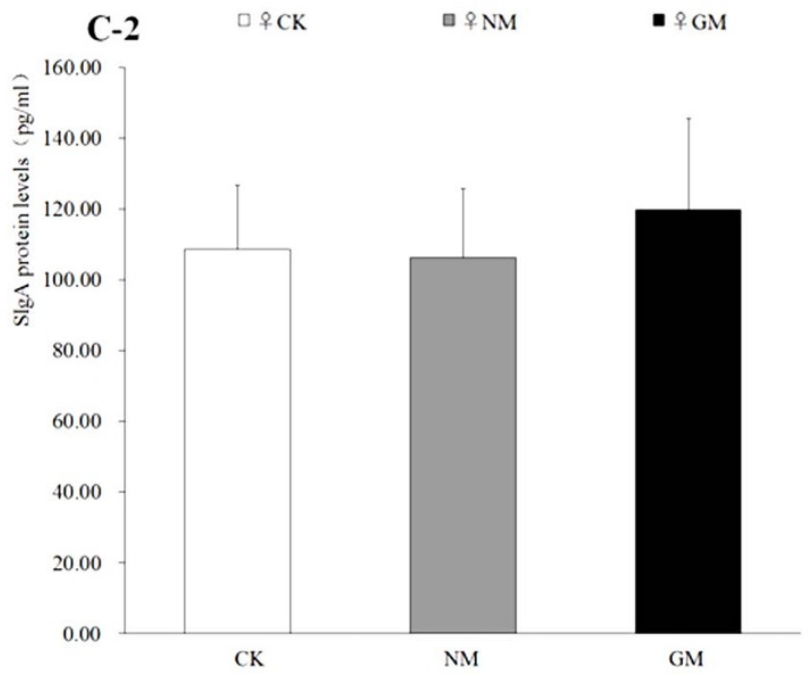

Figure 3 Effects of T2A-1 on ileum tight proteins: Zo-1 and occludin mRNA expression. Changes in $(A)$ Zo-1, $(B)$ Occludin with $\beta$-actin as a housekeeping gene. $(C)$ sIgA expression with ELISA. Values are means $\pm \mathrm{SD}, \mathrm{n}=5$. Means without a common letter differ significantly $(\mathrm{P}<0.05)$.

the relationship between the changes in intestinal permeability and the development of disease, and it was reported that animals with inflammatory bowel disease (IBD) showed higher levels of serum DAO than healthy controls ${ }^{33}$. However, this experiment showed that there were no unintended effects on intestinal permeability due to the feeding of Cry2A rice. A hallmark of mucosal immunity is secretory $\operatorname{IgA}(\operatorname{sIg} A)$. The presence of $\operatorname{sg} A$ can prevent infection of epithelial host cells, protect the surface of mucus membranes from 

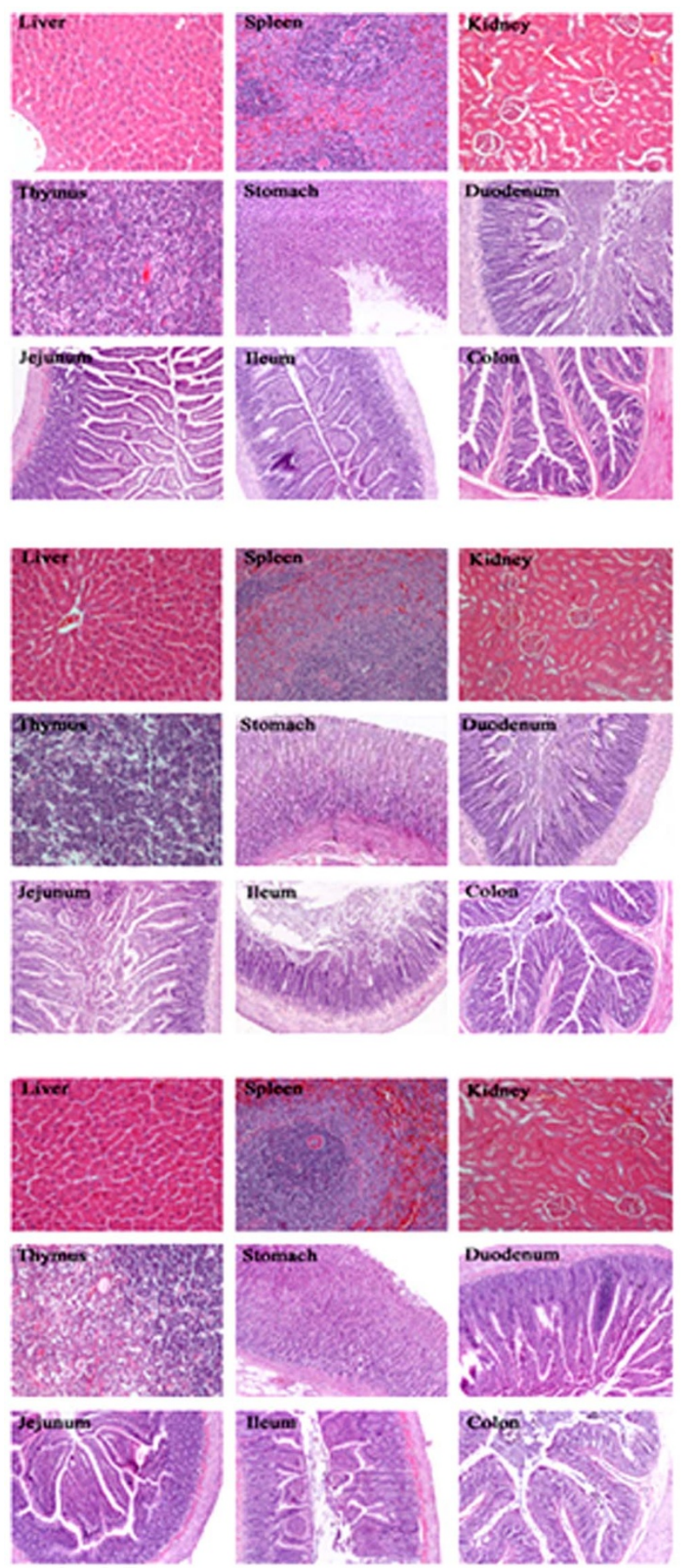

Figure $4 \mid$ Histopathological results. Histopathology results of liver, spleen, kidney, thymus, stomach, duodenum, jejunum, ileum, and colon in (A) AIN93 G group, (B) non-genetically modified (NM) group, and (C) genetically modified (GM) group.

pathogenic infections and carcinogens, and remove antigen that crosses the epithelial barrier by transporting the antigen across the epithelium 34, which plays an important immune function and regulatory role in the GI tract. ELISA results of sIgA expression did not show any difference in the mucosal immunity between GM and NM groups (Fig. 3-C).
Horizontal Gene Transfer (HGT) events occurred in a very low frequency. Despite laborious detection, neither endogenous nor exogenous genes were found in any of the studied tissues (i.e., spleen, kidney, liver, and muscle), GI content, blood, gastric bacterial DNA (or bacterial culture), which reflected a similar degradation of both isogene and transgene, with little possibility for HGT in the GI tract. Recombinant DNA were not detected simultaneously in GI tract, blood or any visceral tissues ${ }^{35}$. Neither transgenic maize nor intrinsic maize fragments were detected in ruminal bacterial culture samples ${ }^{32}$. Einspanier R, et al. could not detect low copy (ivr and zein) or single copy (CryIAb and AmpR) genes in the GI tract of cows fed with Bt176 corn, but detected the presence of high-copy chloroplast genes in ingesta from different GIT sections ${ }^{14}$. The only evidence of gene transfer from GM-soya to gut microorganisms was reported in DNA cultures of human intestinal bacteria ${ }^{23}$. Gene flow from rice to wild rice ${ }^{36}$, but not into soil bacteria has been reported. In this study, the results showed there were no differences between endogenous and exogenous gene with respect to survival in GI tract, suggesting the possibility of HGT is very rare.

Histopathological finding showed no adverse results. No deformations were found in histopathological examinations of the stomach, duodenum, jejunum, ileum and colon of rats (Fig. 4). A three-generation feeding study of Bt corn on rats supports our finding, with no histopathological abnormalities in the mucosa of the small intestine reported ${ }^{37}$. Histomorphological changes have been widely used to assess the effects of GM ingredients on the diets of mice and rats $^{32,38}$. In addition to the GIT, the liver and kidneys are also key metabolic organs responsible for biotransformation, and have important roles in response to toxicants and immune responses, the changes of which suggest alterations in the metabolic processes. A feeding study in rats with Mon 863 Bt corn demonstrated increased inflammation in the liver and kidneys ${ }^{37,38}$. In the 90-day study, male rats fed with 33\% Mon $863 \mathrm{Bt}$ corn had lower kidney weight and some kidney tubular changes. However, our study revealed no abnormal histopathological differences in the kidneys and liver between GM and NM groups.

\section{Discussion}

The global population is steadily growing, while the amount of arable land is steadily decreasing. Bt rice could significantly enhance yield and quality, contributing to the doubling of food, leading to substantial less use of broad-spectrum chemical insecticides. At this rate, global food security and safety could be guaranteed in the future ${ }^{5}$. The progress of commercialization of genetically modified Bt rice calls for more comprehensive and advanced assessment technology to provide safety assurance for human health. Although many animal studies have been carried out to assess the safety of GM materials, these studies have less focused on GI health. A food safety assessment of genetically modified T2A-1 rice has been performed by other scientists on composition, nutrition, allergenicity and toxicology analyses in according to the substance equivalence principle, and no adverse results have been found. This study paid more attention on the effects of T2A-1 rice on the GI health of rats.

There are interactive effects with $\mathrm{pH}$ value, SCFA profile and bacteria composition in content from different parts of GI. The fermentation of carbohydrates by intestinal anaerobic bacteria leads to the production of a spectrum of organic acids including SCFA (mainly acetate, propionate and butyrate) and other gases (e.g., carbon dioxide and hydrogen), which have effects on the $\mathrm{pH}$ value of contents from GIT. And the changes of $\mathrm{pH}$ in the GIT environment will also have effects on the composition of gastric bacteria. In addition, different bacteria composition will in turn have impacts on $\mathrm{pH}$ value and SCFA profile ${ }^{15}$. In this study, no differences were found between GM and NM group in the bacteria composition in content 
from different part of GI tract and in feces at different stages during a 90-day experiment. Higher amounts of Lacotobacillus and Enterococcus in rice control groups than AIN93 G group were supported by lower $\mathrm{pH}$ values and higher concentrations of acetic acid in the same sample. The good relationships among the parameters above have suggested the availability of this model in safety assessment.

Microflora in the GI tract have developed a number of protective, immune and metabolic functions, which altogether have an enormous impact on the nutrition and health status of the host. The metabolic activity developed by the gut microbiota contributes to the digestion of dietary compounds, salvage of energy, supply of (micro) nutrients and transformation of xenobiotics ${ }^{15}$, which also influences epithelial metabolism, proliferation, survival, and barrier function ${ }^{39}$. A dysbiotic microbiota is an ecological disorder of the bacterial community and is often associated with the pathogenesis of $\mathrm{IBD}^{40,41}$. Overall, a balanced gut microbiota composition benefits to the host, while microbial imbalances are associated with metabolic and immune-mediated disorders ${ }^{42}$. Some published studies ${ }^{10,27,28}$ have found changes in the bacteria composition in feces due to the feeding of GM rice and no adverse histopathological apperance. Due to the lack of sufficient data on GI health, these findings are overly simplified and superficial, and may not reflect the whole status of the GI tract. It has been suggested that if changes in microbiota composition and effects on gastric health are supported by a plausible mechanism (e.g., metabolic or immune alterations), their relationships will be considered causal. Some minor damages may be omitted, which may potentially be a threat to gastric and host health, but not strong enough to result in morphological changes in organs or tissues.

SCFAs are not only well-known energy sources for the colonic epithelium and the host, but also have been noted to have immunomodulatory effects by suppression of inflammatory cytokine secretion, and contribute to mucosal tolerance and gut physiological function $^{15}$. Gut epithelium constitutes a physical barrier that regulates the transcellular and paracellular transit of exogenous substances and that impairs the entry of most luminal antigens, which play an important role in host immunity ${ }^{43,44}$. Over the past decade, there has been increasing recognition of an association between disrupted intestinal barrier function and the development of autoimmune and inflammatory diseases ${ }^{45}$. In vitro and in vivo animal studies have demonstrated that intestinal permeability is regulated by multiple factors including exogenous factors, epithelial apoptosis, cytokines, and immune cells ${ }^{45}$. In this study, no differences, with respect to gastric permeability epithelial structure, fecal enzymes, bacterial activity, and immunity, have been observed, and no damages were shown in histopathology studies on different parts of the GIT, thus proving a good status of the GIT.

With respect to unintended effects of HGT on gastric bacteria, there is evidence supporting the transfer of the Bt gene into its relative plants ${ }^{36}$, but the HGT of exogenous genes into tissue and intestinal microbes is still controversial resulting from food for eating or feeding ${ }^{23}$. It has been reported that microbes in the small bowel of ileostomists are capable of acquiring and harboring DNA sequences from GM plants, propeling a great progress in the study of HGT in humans. However, ileostomists in experimental studies may not represent healthy people with intact digest systems, and thus, this conclusion creates more concern. The current research and technology has not met the need of people who are concerned with the unintended effects of GM food. Thus, a good model for the safety assessment of HGT and other unintended effects of GM is essential. Rats have been widely used as a cheap model in safety assessments of food and feed from biotechnology-derived crops $^{46}$.

GI health is a complicated notion and is influenced by many interactive factors. Studies on GI health have focused on the activity of fecal enzymes ${ }^{39,40}$, metabolism products ${ }^{41}$, epithelium structure ${ }^{47}$, and intestinal mucosal immunity ${ }^{39,48}$. All of these factors are connected with each other to protect the equilibrium of the gastric ecosystem. Feces is an important parameter in both clinic and nutritional studies, and offers some advantages such as easy for collection, lack of pain infliction during collection and convenience for longitudinal comparisons. Some changes in feces can give useful information and cues about chronic disease and the nutrition status of host. In this study. We did not investigate urine samples, because it is easy to be contaminated and is restricted to small molecular substance analysis.

This paper presented a systematic approach based on a traditional subchronic toxicity study, with a range of combined parameters including clinical, hematological, histopathological, immunological, microbiological and metabolic parameters. In addition, this study focused on gastric health by trying to establish a systematic model for the safety assessment of unintended effects on the intestinal health of rats. This study is an elementary exploration, which may supply a tool for a well-founded risk-estimation and may provide detailed information about the effects of GM materials on the gastric health of human and animals that would consume food and feed derived from these biotechnology-derived crops.

\section{Methods}

Rice materials. The target rice in this study is the genetically modified insect-resistant rice T2A-1 with a synthetic Cry $2 \mathrm{~A}^{*}$ gene introduced into the elite indica rice restorer line Minghui 63 by Agrobacterium-mediated transformation provided by Huazhong Agricultural University.

Animals and diets. All experimental procedures involving animals were approved by the Ethics Committee of Peking Medical College University. Specific pathogen-free (SPF) Sprague-Dawley (SD) rats at the age of three weeks were kept in accordance with institutional guidelines (Animal Center of Peking University). Rats were housed three per polypropylene plastic cage in a temperature controlled room $\left(21 \pm 2^{\circ} \mathrm{C}\right)$ with a relative humidity of $50 \pm 10 \%$ and a 12 h light-dark cycle. Food and sterilized water were consumed freely throughout the experimental period. Body weights and food intake were recorded weekly. After a one-week acclimatization period, 84 animals (half male and half female) were randomly divided into 14 groups (six rats/ group) according to weight. The rats were assigned to consume AIN93G diet or diets mixed with maximum addition of $70 \%$ Cry $2 \mathrm{~A}$ rice and $70 \%$ parent line rice according to the AIN93G diet. For microbiological analysis, fresh fecal samples were taken at day 30, 60 and 90. At the termination of the study, rats were euthanized using $\mathrm{CO}_{2}$ asphyxiation. Blood was withdrawn just before euthanization for blood biochemistry and hematology analysis. Then, a complete necropsy was performed. Small pieces of selected organs (in detail in the Gross necropsy and histopathology section) were collected for histopathology analysis. Content from the GIT including duodenum, jejunum, ileum, cecum and colon were collected, quick-frozen in liquid nitrogen and kept at $-80^{\circ} \mathrm{C}$ until analysis.

Gross necropsy and histopathology. A complete necropsy was performed, and the following organs were excised and weighed: heart, liver, spleen, lung, kidneys (paired), ovaries (paired), testis (paired), adrenals (paired), thymus, stomach, duodenum, jejunum, ileum, cecum and colon. Sections from the above organs were fixed in $4 \%$ buffered formaldehyde more than $24 \mathrm{~h}$ before histological processing. Tissue samples were embedded in paraffin, and 4-6 $\mu \mathrm{m}$ thick sections were stained with hematoxylin-eosin (H\&E) for light microscopy (OLYMPUS, BX-50, Japan). All tissue sections were observed and analyzed by a Leica DFC300FX. The main focus of the histopathology examinations was on the intestinal tract and related organs. The following tissues were photographed: stomach, duodenum, jejunum, ileum, colon, liver and kidney.

Blood biochemistry and hematology analysis. The following biochemical parameters were measured with an RA-1000 auto-analyzer (Technicon, Tarrytown, NY, USA) including alanine ALT, AST, alkaline phosphatase (ALP), total protein (TP), albumin (ALB), total cholesterol (T-CHOL), triglyceride (TG), creatinine (CREA), glucose (GLUC), blood urea nitrogen (BUN), calcium (Ca) and inorganic phosphorus $(\mathrm{P})$. Hematology characteristics were assessed with a Sysmex F-820 blood cell counter (Kobe, Japan) on the following parameters: white blood cells (WBC), red blood cells (RBC), platelets (PLT), hemoglobin (HGB), hematocrit (HCT), mean corpuscular volume (MCV), MCH and MCHC. The deferential count was performed manually on blood smears for neutrophils $(\mathrm{N})$, lymphocytes $(\mathrm{L})$, eosinophils $(\mathrm{E})$, basophils (B) and monocytes (M).

DNA extraction. DNA was isolated from individual reference bacterial strains at the stationary growth phase with a DNA extraction kit according to the manufacturer's protocol (Cat. A1120, Promega, USA) including Lactobacillus salivarius (CGMCC 2263), Bifidobacterium longum (CGMCC 2265), Enterococcus faecalis (CGMCC 1.125), Escherichia coli (CGMCC 1.90) and Clostridium butyricum (CGMCC 1.209). Culture condition was chosen as described by Jean-Marc Delroissea ${ }^{49}$ and T. 
Rinttila ${ }^{50}$. Feces or different parts of the GI tract were taken for DNA extraction with a stool extraction mini kit (Qiagen, Germany). DNA from the diet sample $(80 \mathrm{mg})$, rice sample $(80 \mathrm{mg})$, tissues samples $(25 \mathrm{mg})$, blood sample $(200 \mu \mathrm{l})$ was extracted using two commercial kits (NucleoSpin Plant and Tissue, Germany). DNA with ratios of OD260/OD280 ranging from 1.8-2.0 was taken for quantification.

Analysis of fecal microflora by real-time quantification PCR method. The realtime PCR analysis was performed with a quantification-PCR core kit (Toyobo) by an ABI Prism SDS 7500 instrument (Applied Biosystem) for analysis of the shift and composition of gastric bacteria (Lactobacillus, Bifidobacterium, Bacteroides, Coliforms, Enterococcus and Clostridium). Standard curves were established using a previously described method ${ }^{45}$ with a detection range from $10^{10}$ to $10^{2}$ DNA copies/ $\mu \mathrm{l}$. The amount of specific bacteria was determined by interpretation of the threshold cycle values to the standard curve, and the results were expressed as $\log 10 \mathrm{CFU} / \mathrm{g}$ feces. Primer sequences targeting the $16 \mathrm{~S} \mathrm{rDNA}$ of different groups or genera of bacteria and total bacteria are listed in Table S6, as previously optimized and described $^{45-50}$

DGGE method for analysis of the bacteria profile. The V3 region of the 16S rRNA gene for all bacteria was amplified with 518R and GC-338F primer pair (Table S6), as described by de Araújo et al. ${ }^{51}$ and Cani et al..$^{52}$. Denaturing Gradient Gel Electrophoresis was performed as described elsewhere ${ }^{52}$. Images were recorded by a GelDoc UV gel documentation system (BioRad, Hercules, CA, USA). Clustering analysis was performed using the unweighted pair group method with arithmetic mean clustering algorithm (UPGMA) to calculate the dendrogram of DGGE gels. Representative bands were excised and sequencing was performed on an ABI PRISM 377 sequencer (Perkin-Elmer). Homology searches of the GenBank DNA database were performed with BLAST (www.ncbi.nlm.nih.gov/BLAST).

Horizontal gene transfer detection detection. Bacteria DNA from different part of GI and DNA from different tissue were used in HGT detection. Primer pairs (Table S6) were designed for detection survival of flanking sequences and exogenous gene sequence (35S, NOS, Cry2A and Bar). The SPS gene was chosen as a rice housekeeping gene, and the chloroplast gene was taken for detection of a plant gene. Real-time PCR was carried out with the SYBR Green technique.

Evaluation of microbial activity. Microbial activities were determined by the content of fecal and cecum SCFA, including acetate, propionate and butyrate, by gas chromatography (GC) as previously described with small changes ${ }^{53,54}$. An aliquot of $200 \mathrm{mg}$ per sample was weighed and suspended in $4 \mathrm{ml} 0.9 \% \mathrm{NaCl}$, homogenized by a chilled motor and then kept at $-20^{\circ} \mathrm{C}$ overnight. Mixtures were centrifuged for $15 \mathrm{~min}$ at $2655 \mathrm{~g}$ at $-4^{\circ} \mathrm{C}$ temperature and $200 \mu \mathrm{l}$ of supernatant was collected and added to $20 \mu \mathrm{l}$ of 4 -methylvaleric acid ( $32 \mathrm{mmol} / \mathrm{l}$, Sigma Chemical Co., St. Louis. MO, USA) and $20 \mu \mathrm{l}$ of $20 \% \mathrm{H}_{3} \mathrm{PO}_{4}$. The samples were mixed well and centrifuged for $10 \mathrm{~min}$ at $17949 \mathrm{~g}$ at $-4^{\circ} \mathrm{C}$. The supernatants were collected and filtered by a filter membrane with a diameter of $2 \mu \mathrm{m}$, and $1 \mu \mathrm{l}$ of the extracts were injected into a gasliquid chromatograph (Hewlett Packard 5890 Series II with a flame ionization detector). A standard SCFA mixture containing acetate, propionate and butyrate (Sigma Chemical Co., St. Louis, MO, USA) was used for calculation, which was assayed before each stool sample. The concentration of SCFA was expressed as mol/g wet weight. Nitrogen was used as the carrier gas. The GC settings were as follows: an initial oven temperature at $80^{\circ} \mathrm{C}$ for $1 \mathrm{~min}$, then increased at $10^{\circ} \mathrm{C} / \mathrm{min}$ to $150^{\circ} \mathrm{C}$, and retained for $10 \mathrm{~min}$. The detector temperature was set at $300^{\circ} \mathrm{C}$. The peak retention time and peak area was recorded.

Measurement of enzyme activity, $\mathrm{pH}$ value and weight of content from feces and cecum. On the last 3 days of the experimental stage, the rats were placed in metabolic cages individually, and fecal output was measured for $48 \mathrm{~h}$. The cecum were excised, measured, weighed and cut open at the end of the experiment. The $\mathrm{pH}$ analysis and water content of fecal sample was measured, as described before ${ }^{55}$, with approximately $0.2 \mathrm{~g}$ of sample. Extra content from fecal samples and cecum were stored at $-80^{\circ} \mathrm{C}$ for later analysis of SCFA concentrations. For enzymes analysis, about $1 \mathrm{~g}$ of fecal sample was transferred into a pre-weighed tube containing $0.1 \mathrm{M}$ phosphate-buffered saline ( $\mathrm{pH} 7.0$ ), the sample was mixed well, sonicated for $30 \mathrm{~s}$, centrifuged at 2,000 g for $5 \mathrm{~min}$, and aliquots of the supernatant were used immediately. Activities of $\beta$-glucuronidase, $\beta$-glucosidase, nitroreductase and $\beta$ galactosidase were measured and reported as milligrams of $\beta$-nitrophenol liberated/ $\mathrm{min} / \mathrm{g}$ protein or micromoles of $\beta$-aminobenzoic liberated $/ \mathrm{min} / \mathrm{g}$ protein.

RNA isolation, reverse transcription and quantification. Total RNA was isolated from ileum and jejunum with a Trizol (Life Technologies) kit according to the manufacturer's instructions and was then reverse transcribed into cDNA with moloney murine leukemia virus reverse transcriptase (Promega, USA) according to the manufacturer's instructions. The real-time PCR method based on SYBR Green I dye was performed for quantification. Details about the primer pair used are shown in Table S6, and $\beta$-actin was used as quality control.

Intestinal permeability analysis. Intestinal permeability in vivo was detected by plasma absorption of 4,000 Da fluorescent dextran-FITC, as previously published ${ }^{52}$. DAO levels in serum were analyzed as previously described ${ }^{56}$. RNA analysis accompanied with an ELISA method for detection of the two proteins $\mathrm{ZO}-1$ and occludin in jejunum was performed as described by Cani et al. ${ }^{39}$. Primer details are available in Table $\mathrm{S} 6$

Mucosal immunofluorescence analysis. The number of IgA secreting cells was determined in histological sections of the rat ileum by a direct immunofluorescent assay as previously described ${ }^{34}$ with a Rat ELISA Quantitation Kit (R\&B Insitute Inc., USA) according to the manufacturer's instructions.

Statistical analysis. Statistical comparisons were conducted to determine whether significant differences were attributed to consumption of diets with T2A-1 rice. For each sex, combined group data variances were analyzed using Levene's test. A oneway analysis of variance (ANOVA) was applied to evaluate the homogeneity variance, and a least squared differences model was conducted to detect the differences in variables using Statistical Product and Service Solutions (SPSS) v12.0 (SPSS Inc., Chicago, IL, USA) statistical software. Data are expressed as mean \pm standard error (SE). Differences were considered significant at $\mathrm{p}<0.05$.

1. Mendelsohn, M., Kough, J., Vaituzis, Z. \& Matthews, K. Are Bt crops safe? Nat Biotechnol 21, 1003-1009 (2003).

2. Betz, F. S., Hammond, B. G. \& Fuchs, R. L. Safety and advantages of Bacillus thuringiensis-protected plants to control insect pests. Regul Toxicol Pharmacol 32, 156-173 (2000).

3. Usda. Plant Health Inspection Service, Veterinary Services, $<$ www. aphis. usda. gov/animal_health/vet_biologics.> (2007).

4. Kennedy, G. G. Integration of insect-resistant genetically modified crops within IPM programs. Integration of Insect-Resistant Genetically Modified Crops within IPM Programs, 1-26 (2008).

5. James, C. Global status of commercialized biotech/GM crops: 2009. ISAAA brief 41 (2009).

6. Ye, R. et al. Development of insect-resistant transgenic rice with Cry1C*-free endosperm. Pest Manag Sci 65, 1015-1020 (2009).

7. Siegel, J. P. The mammalian safety of Bacillus thuringiensis-based insecticides. I Invertebr Pathol 77, 13-21(2001).

8. McClintock, J. T., Schaffer, C. R. \& Sjoblad, R. D. A comparative review of the mammalian toxicity of Bacillus thuringiensis-based pesticides. Pesticide Science 45, 95-105 (1995).

9. Kroghsbo, S. et al. Immunotoxicological studies of genetically modified rice expressing PHA-E lectin or Bt toxin in Wistar rats. Toxicology 245, 24-34 (2008).

10. Schroder, M. et al. A 90-day safety study of genetically modified rice expressing Cryl Ab protein (Bacillus thuringiensis toxin) in Wistar rats. Food Chem Toxicol 45, 339-349 (2007)

11. Vazquez-Padron, R. I. et al. Cry1Ac protoxin from Bacillus thuringiensis sp. kurstaki HD73 binds to surface proteins in the mouse small intestine. Biochem Biophys Res Commun 271, 54-58 (2000).

12. Sagstad, A. et al. Evaluation of stress- and immune-response biomarkers in Atlantic salmon, Salmo salar L., fed different levels of genetically modified maize (Bt maize), compared with its near-isogenic parental line and a commercial suprex maize. J Fish Dis 30, 201-212 (2007).

13. Noverr, M. C. \& Huffnagle, G. B. Does the microbiota regulate immune responses outside the gut? Trends Microbiol 12, 562-568 (2004).

14. Einspanier, R. et al. Tracing residual recombinant feed molecules during digestion and rumen bacterial diversity in cattle fed transgene maize. Eur Food Res Technol 218, 269-273 (2004).

15. Laparra, J. M. \& Sanz, Y. Interactions of gut microbiota with functional food components and nutraceuticals. Pharmacol Res 61, 219-225 (2010).

16. Nissen, L., Chingwaru, W., Sgorbati, B., Biavati, B. \& Cencic, A. Gut health promoting activity of new putative probiotic/protective Lactobacillus spp. strains: a functional study in the small intestinal cell model. Int J Food Microbiol 135, 288294 (2009).

17. Tudisco, R. et al. Genetically modified soya bean in rabbit feeding: detection of DNA fragments and evaluation of metabolic effects by enzymatic analysis. ANIMAL SCIENCE-GLASGOW THEN PENICUIK 82, 193 (2006).

18. Zhou, J. et al. Metabolic profiling of transgenic rice with cryIAc and sck genes: an evaluation of unintended effects at metabolic level by using GC-FID and GC-MS. J Chromatogr B Analyt Technol Biomed Life Sci 877, 725-732 (2009).

19. Heritage, J. Transgenes for tea? Trends Biotechnol 23, 17-21 (2005).

20. Heritage, J. The fate of transgenes in the human gut. Nat Biotechnol 22, 170-172 (2004).

21. Tony, M. A. et al. Safety assessment of Bt 176 maize in broiler nutrition: degradation of maize-DNA and its metabolic fate. Arch Tierernahr 57, 235-252 (2003).

22. Reuter, T., Aulrich, K., Berk, A. \& Flachowsky, G. Investigations on genetically modified maize (Bt-maize) in pig nutrition: chemical composition and nutritional evaluation. Arch Tierernahr 56, 23-31 (2002).

23. Netherwood, T. et al. Assessing the survival of transgenic plant DNA in the human gastrointestinal tract. Nat Biotechnol 22, 204-209 (2004).

24. van den Eede, G. et al. The relevance of gene transfer to the safety of food and feed derived from genetically modified (GM) plants. Food Chem Toxicol $\mathbf{4 2}$, $1127-1156$ (2004).

25. Kelly, B. G., Vespermann, A. \& Bolton, D. J. Gene transfer events and their occurrence in selected environments. Food Chem Toxicol 47, 978-983 (2009). 
26. Kuiper, H. A., Kleter, G. A., Noteborn, H. P. \& Kok, E. J. Assessment of the food safety issues related to genetically modified foods. Plant J 27, 503-528 (2001).

27. Poulsen, M. et al. A 90-day safety study in Wistar rats fed genetically modified rice expressing snowdrop lectin Galanthus nivalis (GNA). Food Chem Toxicol 45, 350-363 (2007).

28. Poulsen, M. et al. Safety testing of GM-rice expressing PHA-E lectin using a new animal test design. Food Chem Toxicol 45, 364-377 (2007).

29. Guarner, F. \& Malagelada, J. R. Gut flora in health and disease. Lancet 361, 512-519 (2003).

30. Cohen, M., Chen, M., Bentur, J. S., Heong, K. L. \& Ye, G. in Integration of InsectResistant Genetically Modified Crops within IPM Programs Vol. 5, Progress in Biological Control (eds Jörg, Romeis, Anthony, M. Shelton \& George, G. Kennedy) Ch. 8, 223-248 (Springer Netherlands, 2008).

31. Xu, W. et al. Safety assessment of Cry1 Ab/Ac fusion protein. Food Chem Toxicol 47, 1459-1465 (2009).

32. Trabalza-Marinucci, M. et al. A three-year longitudinal study on the effects of a diet containing genetically modified Bt176 maize on the health status and performance of sheep. Livestock Science 113, 178-190 (2008).

33. Song, W. B. et al. Soluble intercellular adhesion molecule-1, D-lactate and diamine oxidase in patients with inflammatory bowel disease. World J Gastroenterol 15, 3916-3919 (2009)

34. Paturi, G., Phillips, M., Jones, M. \& Kailasapathy, K. Immune enhancing effects of Lactobacillus acidophilus LAFTI L10 and Lactobacillus paracasei LAFTI L26 in mice. Int J Food Microbiol 115, 115-118 (2007).

35. Rizzi, A. et al. The stability and degradation of dietary DNA in the gastrointestinal tract of mammals: implications for horizontal gene transfer and the biosafety of GMOs. Crit Rev Food Sci Nutr 52, 142-161 (2012).

36. Lu, B. R. \& Yang, C. Gene flow from genetically modified rice to its wild relatives: Assessing potential ecological consequences. Biotechnol Adv 27, 1083-1091 (2009)

37. Kilic, A. \& Akay, M. T. A three generation study with genetically modified Bt corn in rats: Biochemical and histopathological investigation. Food Chem Toxicol 46, 1164-1170 (2008).

38. Seralini, G. E., Cellier, D. \& de Vendomois, J. S. New analysis of a rat feeding study with a genetically modified maize reveals signs of hepatorenal toxicity. Arch Environ Contam Toxicol 52, 596-602 (2007).

39. Hooper, L. V. Bacterial contributions to mammalian gut development. Trends Microbiol 12, 129-134 (2004).

40. Sartor, R. B. Microbial influences in inflammatory bowel diseases Gastroenterology 134, 577-594 (2008).

41. Strober, W., Fuss, I. \& Mannon, P. The fundamental basis of inflammatory bowel disease. J Clin Invest 117, 514-521 (2007).

42. Nadal, I., Donat, E., Ribes-Koninckx, C., Calabuig, M. \& Sanz, Y. Imbalance in the composition of the duodenal microbiota of children with coeliac disease. J Med Microbiol 56, 1669-1674 (2007).

43. Laukoetter, M. G., Bruewer, M. \& Nusrat, A. Regulation of the intestinal epithelial barrier by the apical junctional complex. Curr Opin Gastroenterol 22, 85-89 (2006)

44. Tsukita, S., Furuse, M. \& Itoh, M. Multifunctional strands in tight junctions. Nat Rev Mol Cell Biol 2, 285-293 (2001).

45. Groschwitz, K. R. \& Hogan, S. P. Intestinal barrier function: molecular regulation and disease pathogenesis. J Allergy Clin Immunol 124, 3-20; quiz 21-22 (2009).

46. Hollingworth, R. M. et al. The safety of genetically modified foods produced through biotechnology. Toxicol Sci 71, 2-8 (2003).

47. Resta-Lenert, S. \& Barrett, K. E. Live probiotics protect intestinal epithelial cells from the effects of infection with enteroinvasive Escherichia coli (EIEC). Gut 52, 988-997 (2003).
48. Arrieta, M. C., Madsen, K., Doyle, J. \& Meddings, J. Reducing small intestinal permeability attenuates colitis in the IL10 gene-deficient mouse. Gut 58, 41-48 (2009).

49. Delroisse, J. M. et al. Quantification of Bifidobacterium spp. and Lactobacillus spp. in rat fecal samples by real-time PCR. Microbiol Res 163, 663-670 (2008).

50. Rinttila, T., Kassinen, A., Malinen, E., Krogius, L. \& Palva, A. Development of an extensive set of $16 \mathrm{~S}$ rDNA-targeted primers for quantification of pathogenic and indigenous bacteria in faecal samples by real-time PCR. J Appl Microbiol 97, 1166-1177 (2004).

51. de Araujo, J. C. \& Schneider, R. P. DGGE with genomic DNA: suitable for detection of numerically important organisms but not for identification of the most abundant organisms. Water Res 42, 5002-5010 (2008).

52. Cani, P. D. et al. Changes in gut microbiota control inflammation in obese mice through a mechanism involving GLP-2-driven improvement of gut permeability. Gut 58, 1091-1103 (2009).

53. Beards, E., Tuohy, K. \& Gibson, G. Bacterial, SCFA and gas profiles of a range of food ingredients following in vitro fermentation by human colonic microbiota. Anaerobe 16, 420-425 (2010).

54. Zhao, G., Liu, J. F., Nyman, M. \& Jonsson, J. A. Determination of short-chain fatty acids in serum by hollow fiber supported liquid membrane extraction coupled with gas chromatography. J Chromatogr B Analyt Technol Biomed Life Sci 846, 202-208 (2007).

55. Liu, R. \& Xu, G. Effects of resistant starch on colonic preneoplastic aberrant crypt foci in rats. Food Chem Toxicol 46, 2672-2679 (2008).

56. Yao, Y. M. et al. Role of gut-derived endotoxaemia and bacterial translocation in rats after thermal injury: effects of selective decontamination of the digestive tract. Burns 21, 580-585 (1995).

\section{Acknowledgements}

This work was supported by Genetically Modified Organisms Breeding Major Projects of P.R. China grant 2011ZX08011-005 and 2012ZX08011003.

\section{Author contributions}

Y.Y. designed and performed most experiments, analysed data and wrote the manuscript W.X. designed and performed experiments, analysed data and commented on the manuscript. X.H. performed and analysed experiments, and critically read the manuscript. H.L. advised on experimental design and commented on the manuscript. S.C., X.Q. performed and analysed experiments. K.H. and Y.L. arranged funding, designed experiments, supervised and mentored all work.

\section{Additional information}

Supplementary information accompanies this paper at http://www.nature.com/ scientificreports

Competing financial interests: The authors declare no competing financial interests.

How to cite this article: Yuan, Y.F. et al. FITCEffects of genetically modified T2A-1 rice on the GI health of rats after 90-day supplement. Sci. Rep. 3, 1962; DOI:10.1038/srep01962 (2013).

This work is licensed under a Creative Commons AttributionNonCommercial-NoDerivs Works 3.0 Unported license. To view a copy of this license, visit http://creativecommons.org/licenses/by-nc-nd/3.0 